\title{
The course of gastric cancer following surgery is associated with genetic variations of the interleukin-1 receptor antagonist and interleukin-1 $\beta$
}

\author{
Anke H. van der Ploeg • Oliver Kumpf • Evelyn Seelow • \\ Luis C. Berrocal Almanza - Peter M. Schlag • \\ Ralf R. Schumann · Lutz Hamann
}

Received: 23 April 2013/Accepted: 1 February 2014/Published online: 21 February 2014

(C) The International Gastric Cancer Association and The Japanese Gastric Cancer Association 2014

\begin{abstract}
Background Inflammation, especially the cytokine response of the IL-1 family, has been shown to influence susceptibility to gastric cancer. In addition, several other pro-inflammatory cytokines have been demonstrated to influence metastasis and resistance to chemotherapy. Therefore, genetic variations within these genes may not only affect susceptibility but also influence the outcome of gastric cancer patients. A limited number of studies showed indeed an association of $I L-1 \beta$ and $I L-I R N$ variations with survival of gastric cancer patients. However, results are inconsistent, possibly because of different patient cohorts and different therapies.

Methods In this retrospective cohort study we genotyped 154 patients with gastric cancer for $I L-1 \beta$ and $I L-l R N$ variations. Patients had undergone pathologically proven R0 resection and had received no additional adjuvant treatment.
\end{abstract}

A. H. van der Ploeg and O. Kumpf equally contributed to this work. R. R. Schumann and L. Hamann equally contributed to this work.

The study has been approved by the institutional ethics committee of the Charité University Medicine Berlin (AA3/03/45).

A. H. van der Ploeg - E. Seelow - L. C. B. Almanza ·

R. R. Schumann · L. Hamann $(\bowtie)$

Institute for Microbiology and Hygiene, Charité University

Medicine Berlin, Hindenburgdamm 27, 12203 Berlin, Germany

e-mail: lutz.hamann@charite.de

O. Kumpf

Department of Anaesthesiology and Intensive Care Medicine, Charité University Medicine Berlin, 13453 Berlin, Germany

P. M. Schlag

Charité Comprehensive Cancer Center, Charité University

Medicine Berlin, 10117 Berlin, Germany
Results We show here a protective association with disease-free survival for both heterozygous genotypes, $I L-1 \beta$ SNP C-511T (rs16944) and $I L-1 R N$ VNTR. The combination of both heterozygous genotypes is the strongest predictor independent of UICC stage.

Conclusion Genetic variations in the $I L-1 \beta$ and $I L-1 R N$ genes influence disease progression in gastric cancer. Screening for these genetic variations might help to stratify therapies for gastric cancer patients in the future.

Keywords Innate immune response $I L-I R N$ variable number tandem repeat $\cdot I L-1 \beta$ polymorphism

\section{Introduction}

Gastric cancer is the third most common cause of cancerrelated death for male individuals in the developed world. Despite the decline in incidence, nearly 1 million new cases are diagnosed per year [1]. In general, chronic inflammation is a risk factor for most cancers [2]. One of the main reasons for chronic inflammation of the stomach is colonization with Helicobacter pylori. Subsequent chronic gastritis is the most recognized etiological risk factor for non-cardia gastric cancer [3-5]. Although half the world's population has been infected with $H$. pylori, only $<3 \%$ of the infected subjects are diagnosed with gastric cancer, indicating the strong influence of the host genetic background [6,7]. The immunological response to $H$. pylori infection strongly involves the cytokine response of the innate immune system, and particularly interleukin 1 (IL-1) [8]. In agreement with this, variations within the $I L-1$ gene family have been associated with the susceptibility to gastric cancer $[9,10]$. 
The $I L-1 \beta$ single-nucleotide polymorphisms (SNPs), located within the $I L-1 \beta$ promoter, are closely linked and have been shown to interfere with transcriptional activity of the $I L-1 \beta$ gene [9]. The penta-allelic polymorphism of the $I L-1 R N$ gene (coding for the IL-1 receptor antagonist, IL-1RA) corresponding to variable numbers of a 86-bp sequence in intron 2 and first identified by Tarlow et al. [11], has been shown to alter the inflammatory response. However, there have been conflicting reports regarding the functional consequence of these variations. Although chronic inflammation and, subsequently, genetic variations affecting the immune responses have been well associated with an increased risk for cancer [2, 10], few data regarding their influence on outcome following surgery are available. Because the inflammatory network in cancer not only affects susceptibility but also metastasis and chemoresistance, an impact of the immune status on the course of the disease and prognosis should be expected [2].

Recently, three studies investigated polymorphisms within the $I L-1$ gene cluster and the course of gastric cancer in patients receiving neoadjuvant or palliative chemotherapy [12-14]. Stocker et al. [13] showed the homozygous $I L-1 R N \mathrm{~S}$ allele to be significantly associated with worse outcome in patients receiving neoadjuvant chemotherapy, whereas the $I L-1 \beta$ SNP C-511T (rs16944) is not. Of note, they found no association with chemotherapy response. In contrast, Graziano et al. [12-14] postulated a favorable effect of the $I L-I R N S$ allele by counterbalancing the adverse influence of the $I L-1 \beta \mathrm{C}-511 \mathrm{~T}$ allele in advanced gastric cancer patients receiving palliative chemotherapy. Tahara et al. [14] reported a protective effect of the $I L-1 \beta$ C-511T allele in advanced gastric cancer.

The usual treatment strategy for newly diagnosed gastric cancer includes surgical resection and perioperative chemotherapy according to the disease stage at diagnosis [15]. To avoid the influence of chemotherapy or severity of the disease on genetic association, we here investigated the association of the $I L-1 \beta$ SNP C-511T and $I L-1 R N$ variations with the outcome of 154 gastric cancer patients who all had complete tumor resection (pathologically proven R0 resection) without additional perioperative treatment.

We found here the homozygous wild type and mutated genotype for both $I L-I R N$ and $I L-1 \beta C-511 \mathrm{~T}$ to be associated with shorter disease-free survival. This finding is in partial agreement with Stocker et al. [13], who found the $I L-1 R N \mathrm{~S} / \mathrm{S}$ homozygote mutated $I L-I R N$ genotype to be associated with a worse outcome. In our study, only the heterozygous $I L-1 R N$ genotype showed an improved outcome. Similar results were obtained for the $I L-1 \beta$ SNP C-511T, showing again the heterozygous genotype to be associated with longer diseasefree survival. The combination of both heterozygous genotypes improved the association in a multivariate analysis adjusted for other independent predictors. Apart from the
UICC tumor stage at the time point of diagnosis and Lauren classification, only the genotype was associated with tumor outcome. The protective effect of this genotype seems to act predominantly in diffuse and mixed type of gastric cancer.

\section{Materials and methods}

\section{Patients}

Patients included in this retrospective cohort study were treated at the Robert-Rössle Klinik, Berlin-Buch, Charité Medical Center Berlin between 1999 and 2006. All patients had histologically proven gastric adenocarcinoma that was diagnosed before R0 resection. Surgical procedures included total or partial gastrectomy with D2 lymphadenectomy and additional resection of adjacent organs if surgically necessary for complete resection. Radiologic suspicion of distant metastasis at the time of surgery led to exclusion from the study. Also patients with a previously operated stomach, preoperative neoadjuvant chemotherapy, existence of esophageal or other than non-cardia gastric cancer, no exact tumor location, and unknown UICC stage were excluded from analysis because of possible interference with prognosis. Patients were followed up by the institution's outpatient clinic and study center. All patients gave a broad consent before treatment to participate in the studies. All steps of the study were performed according to the principles of the Helsinki declaration. A total of 154 patients could be included and analyzed for $I L-1 R N$ and $I L-1 \beta$ variations. A summary of the included patient characteristics is given in Table 1.

\section{Genotyping}

Genomic DNA was isolated by standard procedures from whole blood. All subjects were genotyped for the VNTR of $I L-1 R N$ and $I L-1 \beta$ C-511T (rs16944) by polymerase chain reaction (PCR) and gel electrophoresis or melting curve analysis, respectively.

$I L-I R N$ variations were determined using primers (TIB Molbiol, Berlin, Germany) described by Tarlow et al. [11]. The PCR conditions were as follows: $95{ }^{\circ} \mathrm{C}$ for $4 \mathrm{~min}$; then 30 cycles of $94{ }^{\circ} \mathrm{C}$ for $30 \mathrm{~s}, 60^{\circ} \mathrm{C}$ for $30 \mathrm{~s}$, and $72{ }^{\circ} \mathrm{C}$ for $40 \mathrm{~s}$; then $72{ }^{\circ} \mathrm{C}$ for $5 \mathrm{~min}$. The PCR products were analyzed by gel electrophoresis. The alleles were named as described by Tarlow et al. [11], whereby alleles 1, 2, 3, 4, and 5 consist of four, two, five, three, and six 86-bp repeats, respectively. The alleles 1 and 2, also known as long and short, respectively, represent the majority of alleles.

$I L-1 \beta$ genotyping for the $I L-1 \beta$ C-5211T (rs16944) SNP was performed by melting curve analysis employing the LightCyler LC 480 platform (Roche Diagnostic, Mannheim, Germany) using the following primers and probes: 
Table 1 Patient characteristics of the 154 patients in the study

\begin{tabular}{ll}
\hline Characteristic & $93(60.4)$ \\
Male sex, number (\%) & $62.0(12.1)$ \\
Mean age, years (SD) & \\
Histopathological features & \\
UICC number (\%) & $27(17.5)$ \\
Stage IA & $30(19.5)$ \\
Stage IB & $36(23.4)$ \\
Stage IIA & $35(22.7)$ \\
Stage IIB & $17(11.0)$ \\
Stage IIIA & $8(5.2)$ \\
Stage IIIB & $1(0.6)$ \\
Stage IIIC & \\
Lauren, number $(\%)^{\mathrm{a}}$ & $52(33.8)$ \\
Intestinal & $67(43.5)$ \\
Diffuse & $17(11.0)$ \\
Mixed & $34(22.1)$ \\
Early gastric cancer & \\
Surgical procedures & $115(74.7)$ \\
Gastrectomy & $14(9.1)$ \\
Distal resection $(2 / 3$ or $4 / 5)$ & $25(16.2)$ \\
Cardia resection & $38(24.7)$ \\
Multivisceral procedure & \\
\hline
\end{tabular}

${ }^{a}$ Patients without Lauren classification, $n=18$ (11.7\%): missing or impossible classification. Multivisceral procedure: any gastric resection in combination with adjacent organs as necessary for complete tumor removal

forward primer: tggcattgatctggttcatc; reverse primer: caatagccctcctgtctgtat, anchor probe: LC640-aacagcaccca aggtagagacccacacc, and sensor probe acagagagctccegag gcaga-FL, resulting in melting peaks at $65.3^{\circ} \mathrm{C}$ and $57.6{ }^{\circ} \mathrm{C}$ for the wild type and mutated allele, respectively. The LightCycler assay was designed by O. Landt (TIBMOLBIOL, Berlin, Germany).

\section{Statistical analysis}

Variables influencing disease-free survival were analyzed by univariate and multivariate backward Cox regression employing the IBM SPSS Statistics software package (version 20.0; IBM, Munich, Germany). Values of $P<0.05$ were considered statistically significant.

\section{Results}

Characteristics of patients: short- and long-term outcome

Overall, 154 patients were included in the analysis. Mean age at diagnosis was 62.0 years (SD, 12.1); 93 patients were male $(60.4 \%)$ and 61 were female (39.6\%), which roughly represents epidemiological data on prevalence in Germany [16]. All patients underwent complete surgical resection with curative intention. Most of the procedures were complete gastrectomies $(n=115,74.7 \%)$ or resection of the cardia $(n=25,16.2 \%)$, in part with adjacent organs, then considered as multivisceral resections $(n=38,24.7 \%)$. In 14 patients $(9.0 \%)$, distal stomach resection was performed. In all patients the described surgery resulted in a histological proven complete removal of the tumor (R0 resection). No perioperative death occurred in this cohort. Patients were further classified into UICC classes (UICC Version 2007): 34 patients (22.1\%) had early gastric cancer. Table 1 gives an overview of the patient characteristics in the study.

Overall mortality in the study period was $77 / 154$ $(50.0 \%)$ : 58 of these deaths $(75.3 \%)$ were gastric cancer related and 16 were not tumor related $(20.8 \%)$ or were a newly diagnosed secondary malignity $(n=3,3.9 \%)$. The 5 -year mortality was $41.6 \%(n=64)$. Mean survival for the cohort was 43.7 months (95\% CI, 40.5-47.0). In 71 patients, the tumor recurred (5 local recurrence, 53 local or distant metastasis, and 13 both). Tumor recurrence correlated with UICC classes, and mortality from cancer occurred more frequently in higher UICC classes. We therefore regarded this cohort as representative of operated gastric cancer patients in general.

\section{Results of genotyping}

Overall, we describe two genetic variations in the $I L-1$ gene family. As described, the $I L-I R N$ VNTR consists of five major alleles resulting in several possible genotypes. In our study $150(97.5 \%)$ of the patients were carrying one of the most common genotypes, L/L (79/150), L/S (60/150), or $\mathrm{S} / \mathrm{S}(11 / 150)$. The frequency of the long allele was $73 \%$. Overall, four rare genotypes, of which only allele 3 was present in this cohort, were detected.

The $I L-1 \beta$ C-511T SNP was present in 74 patients (53.9\%), of which 19 were homozygous and 65 heterozygous. Thus, the allele frequency was $33 \%$, which is consistent with a previously reported distribution [9]. The distribution of these genotypes is shown in Table 2. Both variations were in Hardy-Weinberg equilibrium.

Risk assessment for disease-free survival

To estimate the effect of single risk factors on disease-free survival, we calculated hazard ratios (HR) for different factors potentially being associated by univariate Cox regression analysis (Table 3). Disease-free survival was defined as absence of metastasis and tumor recurrence. As expected the known risk factors were UICC stage [IB: HR, 
Table 2 Distribution of $I L-1 R N$ and $I L-1 \beta-511$ genotypes

\begin{tabular}{ll}
\hline Variant & Number $(\%)$ \\
\hline$I L-1 R N^{\mathrm{a}}$ & $n=150$ \\
$\mathrm{~L} / \mathrm{L}(1 / 1)$ & $79(51.3)$ \\
$\mathrm{L} / \mathrm{S}(1 / 2)$ & $60(39.0)$ \\
$\mathrm{S} / \mathrm{S}(2 / 2)$ & $11(7.3)$ \\
$I L-1 \beta-511$ & $n=154$ \\
Wild-type C/C & $70(45.5)$ \\
Heterozygote C/T & $65(42.2)$ \\
Homozygote T/T & $19(12.3)$ \\
\hline
\end{tabular}

${ }^{\mathrm{a}}$ For the IL-1RN variant, only common genotypes were included (3 patients displayed the $1 / 3$ and 1 patient the $2 / 3$ genotype)Therefore, patient number is $n=150$. $L$ long, $S$ short. Both variations were in Hardy-Weinberg equilibrium $(P=0.93$ and $P=0.52$, respectively)

Table 3 Univariate Cox regression analysis for disease-free survival

\begin{tabular}{llcl}
\hline Predictor & $P$ value & Exp. $\beta(\mathrm{HR})$ & $95 \%$ CI for HR \\
\hline UICC stage & & & \\
IA & Ref. & & \\
IB & 0.46 & 1.36 & $0.60-3.05$ \\
IIA & 0.001 & 3.31 & $1.60-6.85$ \\
IIB & $<0.001$ & 3.79 & $1.83-7.86$ \\
IIIA & $<0.001$ & 8.74 & $3.89-19.62$ \\
IIIB & $<0.001$ & 11.17 & $4.28-29.18$ \\
IIIC & 0.116 & 5.26 & $0.67-41.60$ \\
Lymphatic invasion $^{\mathrm{a}}$ & $<0.001$ & 2.31 & $1.56-3.43$ \\
Early gastric cancer & \\
Gender & $<0.001$ & 3.27 & $1.83-5.87$ \\
Lauren classification $^{\mathrm{c}}$ & 0.082 & 0.70 & $0.47-1.05$ \\
IL1-RN $^{\mathrm{d}}$ & 0.005 & 0.56 & $0.37-0.84$ \\
IL1- $\beta$ C-511T $^{\mathrm{d}}$ & 0.054 & 0.67 & $0.44-1.01$ \\
IL1- $\beta$ C-511T*IL1-RN $^{*}$ & 0.010 & 0.51 & $0.31-0.85$ \\
\hline
\end{tabular}

Reference categories: ${ }^{\mathrm{a}}$ Yes, ${ }^{\mathrm{b}}$ Male, ${ }^{\mathrm{c}}$ Intesinal, ${ }^{\mathrm{d}}$ Heterozygous

1.36, $P=0.46$; IIA: HR, $3.31, P=0.001$; IIB: HR, 3.79 , $P<0.001$; IIIA: HR, $8.74, P<0.001$; IIIB: HR, 11.17 , $P<0.001$; and IIIC: HR, 5.26, $P=0.116$ (because there is only one patient with UICC IIIC this $P$ value is not significant], lymphatic invasion (HR, 2.31, $P<0.001$ ), early gastric cancer (HR, 3.27, $P<0.001$ ), and Lauren classification (intestinal versus diffuse and mixed type: HR, $0.56, P=0.005)$ showed a significant association with disease-free survival. As expected, higher UICC stages, lymphatic invasion, and early gastric cancer are associated with increased hazard ratios, whereas intestinal gastric cancer is associated with decreased hazard ratio compared to diffuse and mixed type of gastric cancer. Gender showed only a borderline association, with males having a slightly improved disease-free survival (HR, $0.70, P=0.082)$. Age
Table 4 Multivariate Cox regression analysis for disease-free survival including the interaction variable

\begin{tabular}{llll}
\hline Predictor & $P$ value & Exp. $\beta(\mathrm{HR})$ & $95 \%$ CI for HR \\
\hline UICC stage & & & \\
IA & Ref. & & \\
IB & 0.83 & 0.92 & $0.40-2.09$ \\
IIA & 0.009 & 2.70 & $1.28-5.71$ \\
IIB & 0.001 & 3.38 & $1.59-7.15$ \\
IIIA & $<0.001$ & 6.27 & $2.63-14.93$ \\
IIIB & $<0.001$ & 7.95 & $2.78-22.72$ \\
IIIC & 0.176 & 4.20 & $0.53-33.48$ \\
Lauren & 0.008 & 0.55 & $0.37-0.86$ \\
IL1- $\beta$ C-511T*IL1- & 0.009 & 0.46 & $0.26-0.82$ \\
RN & & & \\
\hline
\end{tabular}

${ }^{a}$ Heterozygous reference category. Other variables in model: lymphatic invasion, early gastric cancer, gender, $I L-1 \beta$ C-511T, IL1 RN

showed no association with disease-free survival (data not shown).

Influence of the genotype on disease-free survival in gastric cancer patients

Univariate Cox regression analysis revealed that, in addition to known risk factors, the heterozygous genotypes of both $I L-1 R N$ VNTR and $I L 1-\beta$ C-511 SNPs are also significantly associated with disease-free survival (Table 3 ). Heterozygous ILI RN VNTR and ILI- $\beta$ C-511 results in improved disease-free survival (HR, $0.67, P=0.054$; HR, $0.67, P=0.045$, respectively). Because there are many conflicting data in the literature regarding the effect of these two genetic variations, we tested whether these two variations may influence each other by analyzing their interaction effect (IL-1-RN*IL-1 $\beta-511)$, comparing patients heterozygous for both variations with patients who were not heterozygous for both. As shown in Table 3 this interaction variable showed a stronger positive effect on disease-free survival than the two variables alone did (HR, $0.51, P=0.010$ ).

UICC stages, Lauren classification, and the combined heterozygous genotype are the strongest predictors for disease-free survival

Employing backward multivariate Cox regression analysis with all variables analyzed previously, we could show that only UICC stage (IB: HR, $0.83, P=0.92$; IIA: HR, 2.70 , $P=0.009$; IIB: HR, $3.38, P=0.001$; IIIA: HR, 6.27 , $P<0.001$; IIIB: HR, 7.95, $P<0.001$; IIIC: HR, 4.20, $P=0.176$ ), Lauren classification (HR, 0.55, $P=0.008$ ), and the combined genotype (HR, 0.46, $P=0.009)$ are significant independent risk and protective predictors, 
Table 5 Multivariate Cox regression analysis for disease-free survival in separate patient groups differing by Lauren type

\begin{tabular}{llll}
\hline Predictor & $P$ value & $\begin{array}{l}\text { Exp. } \beta \\
(\mathrm{HR})\end{array}$ & $\begin{array}{l}95 \% \text { CI } \\
\text { for HR }\end{array}$ \\
\hline $\begin{array}{l}\text { UICC stage } \\
\text { Diffuse type of gastric cancer }\end{array}$ & & \\
IA & Ref. & & \\
IB & 0.865 & 1.09 & $0.41-2.92$ \\
IIA & 0.082 & 2.29 & $0.90-5.81$ \\
IIB & 0.023 & 2.87 & $1.16-7.10$ \\
IIIA & $<0.001$ & 16.68 & $5.57-49.94$ \\
IIIB & 0.006 & 7.04 & $1.76-28.14$ \\
IIIC & 0.129 & 5.17 & $0.62-43.18$ \\
IL1- $\beta$ C-511T*IL1-RN ${ }^{\mathrm{a}}$ & 0.008 & 0.34 & $0.15-0.75$ \\
Intestinal type of gastric cancer & & \\
IA & Ref. & & \\
IB & 0.890 & 0.89 & $0.18-4.23$ \\
IIA & 0.078 & 3.85 & $0.86-17.15$ \\
IIB & 0.040 & 4.98 & $1.08-22.98$ \\
IIIA & 0.102 & 4.22 & $0.75-23.70$ \\
IIIB & 0.007 & 13.28 & $2.04-86.43$ \\
\hline
\end{tabular}

${ }^{a}$ Heterozygous reference category. Other variables in model: lymphatic invasion, early gastric cancer, gender, $I L-1 \beta$ C-511T, IL1 RN

respectively (Table 4). As it is known that intestinal and diffuse/mixed types of gastric cancer rely on different pathomechanisms, we analyzed these two groups separately. As shown in Table 5, the combined genotype is a protective predictor only in patients with diffuse or mixed type of gastric cancer (HR, $0.34, P=0.008$ ) but not in patients with intestinal type of gastric cancer. As expected the UICC stage is a similarly strong risk predictor in both groups.

\section{Discussion}

Genetic variations affecting the immune response have been well associated with the risk of gastric cancer [17]. Variations of the genes encoding for the IL-1 family in particular seem to be associated [9]. It is known that members of the IL-1 family are involved in multiple aspects of tumor biology such as carcinogenesis, metastasis, and angiogenesis (reviewed by Dinarello [18]). Most studies on the effects of IL-1 clearly show that the proinflammatory effects of IL-1 $\beta$ negatively influence tumor biology by increasing tumor growth and by facilitating angiogenesis among others. Furthermore, IL-1 $\beta$ has also been shown to be involved in chemoresistance [19]. Additionally, decreasing effects of the antiinflammatory IL-1 RA also promote tumor growth in several models, including blocking antibodies and knockout mice [18]. In line, pro-inflammatory variations of the IL-1 family have been associated with an increased risk for gastric cancer [9].

Because metastases as well as chemoresistance are prognostic factors for survival, it has been speculated that variations of the IL-1 family genes may also affect patient outcome. To date, only three studies are available investigating $I L-1 \beta$ and $I L-1 R N$ variations and survival of gastric cancer patients, and these led to conflicting results [12-14]. Stocker et al. [13] found the $I L-1 R N$ S/S genotype to be associated with worse outcome, whereas the C-511T IL-1 $\beta$ SNP is not. Garziano et al. [12] and Tahara et al. [14] found the $I L-1 \beta$ promoter variations to be associated with patient survival. These converse results may depend on differences in ethnicity or other patient characteristics or therapies applied. Furthermore, the study groups differed in Lauren classification and received different therapies, neo-adjuvant or palliative, respectively. Of note, despite different results regarding genotype associations, both Stocker and Graziano found the detected associations were not influenced by therapy received $[12,13]$.

We investigated here a highly homogeneous collective of gastric cancer patients. None of them had organ metastasis at the time of diagnosis, all were treated as R0 resection, and none of them received perioperative neoadjuvant or adjuvant chemotherapy. In agreement with Stocker et al. [13], we found the homozygous $I L-I R N$ allele $\mathrm{S}$ to be associated with decreased outcome. In contrast, we also found the homozygous $I L-I R N$ allele $\mathrm{L}$ to be associated with a worse outcome; only the heterozygous L/S genotype showed an improved disease-free survival. In agreement with Garciano et al. [12], we found an association of the $I L-1 \beta$ C-511T variation with survival. Similar to $I L-1 R N$ variations, the heterozygous genotype was significantly associated with improved outcome. In addition, the combination of both heterozygous genotypes showed a further improved survival compared to subjects carrying only one or no heterozygous genotype. Besides UICC staging and Lauren classification, this combined genetic variable is the only independent variable associated with disease-free survival. Furthermore, the protective effect of this genotype seems to act predominately in diffuse and mixed type of gastric cancer. However, because of the low and different sample numbers this result should be taken with care. Larger study groups are needed to confirm this result.

The $I L-1 \beta$ promoter polymorphisms have been associated with increased IL-1 $\beta$ production [9] Also, the IL-IRN $\mathrm{S}$ allele has been shown to be associated with enhanced IL$1 \beta$ production, although conflicting data have been reported $[20,21] . I L-I R N$ and $I L-1 \beta$ variations may affect IL-1 $\beta$ production differentially. Therefore, investigation of single variations may be the reason for conflicting data. In this 
regard, our finding that the $I L-I R N$ and $I L-1 \beta$ variations influence each other is of special interest. However, proinflammatory variations of the IL- 1 family have been associated with an increased risk for gastric cancer and may potentially also be associated with worse outcome after surgery [9].

Our analysis suggests that the protective effect of the heterozygous genotype is predominantly present in patients with diffuse or mixed-type carcinomas. The chain of events leading to these specific subtypes of gastric cancer is different as described in a recent review by Grabsch and Tan [22]. One important step leading to the development of the diffuse type of gastric cancer is hypermethylation of the E-cadherin promoter [23]. There is one report linking the $I L-1 \beta$ C-511T variation with hypermethylation of E-cadherin [24]; however, it seems necessary to further evaluate inflammatory pathways in these distinct histopathological subtypes as this could help us understand the etiology and pathogenesis of these subtypes of gastric cancer.

Our data show a heterozygote advantage for both the $I L$ $1 \beta \mathrm{C}-511 \mathrm{~T}$ SNP and the $I L-1 R N$ VNTR by univariate analysis. A recent meta-analysis on these variations and susceptibility to gastric cancer was able to show an increased risk for $I L-1 \beta$ C-511T TT plus CT (T carriers) versus $\mathrm{CC}$ genotypes based on a dominant model. Furthermore, these authors showed an association of the $I L$ $I R N \mathrm{~S}$ allele with increased risk of gastric carcinoma [25]. These results seem to contradict our findings, but our study investigated survival and long-term effects, and not susceptibility, which could be the reason for different results. Furthermore, the finding that both variations may influence each other could be an explanation for these contradicting results.

However, heterosis has been postulated for several other variations in common genetic diseases, e.g., malaria, and has been postulated to be a natural consequence of adaption in diploids [26, 27]. Regarding the inflammatory response, heterozygosity of the mannose binding lectin genotype predicts an advantage to fatal outcome of sepsis patients [28]. Furthermore, heterozygosity of the Toll-like receptor adaptor protein TIRAP/MAL has been associated with protection from tuberculosis, pneumococcal disease, and bacteremia [29]. Different molecular mechanisms for heterosis are discussed by Comings and MacMurray [30]. One possibility is the involvement of one mutation in different pathomechanisms. In case of sickle cell trait the homozygous mutation within the globin gene is deleterious because of anemia, whereas the wild-type genotype is prone to Plasmodium falciparum infection, resulting in a heterozygote advantage [31].

IL-1 $\beta$ is a pleiotrophic cytokine with a variety of functions [18]. Little is known about short- and long-term regulation of IL-1 $\beta$ in malignant disease. One proposed mechanism of tumor defense even involves Il-1 $\beta$ for eradication of tumors [32]. Also, regulation of IL-1 $\beta$ levels and other pro- or antiinflammatory cytokines of that family might interact, tending toward a certain balance. Also, resistance against endogenous IL- $1 \beta$ may develop, making these carriers less prone to potential negative IL- $1 \beta$ effects. One potential mechanism of reduced tumor recurrence despite potentially higher IL-1 $\beta$ levels in heterozygous carriers of either variant may be caused by a certain resistance to these levels by downregulated receptors or through other regulatory mechanisms. However, this is highly speculative as there is not substantial scientific evidence on this matter. Overall, the effects on cancer are complex and often of opposing nature (reviewed by Apte and Voronov [33]) and may therefore result in heterozygote advantage regarding certain aspects of disease progression.

In conclusion, we show here that both heterozygous genotypes of the $I L-1 R N$ and $I L-1 \beta$ C-511T variation are associated with enhanced disease-free survival. Especially, the combined heterozygote genotype is an independent protective predictor for disease-free survival predominantly in patients with diffuse and mixed-type gastric cancer. This combined genotype has a stronger impact than most other associated factors and is, together with UICC stage and Lauren classification, the only independent predictor of disease-free survival. Although clear data on the local and systemic effects of these variations on IL-1 cytokines are lacking, it is worth speculating that screening for these genetic variations might help tailor therapies involving, for example, direct inhibition of IL-1 or other cytokines involved in the process of tumor growth and spread of metastasis [34]. Furthermore, if our results could be confirmed by larger studies, one may speculate on including genotyping into UICC scoring.

Acknowledgments We thank the patients participating in this study. Financial support was provided by Charite - Universitätsmedizin Berlin (grant 2007-486), the Berliner Krebsgesellschaft e.V., and by the International Graduate School (IRTG) GRK1673 (to R.R.S.). We acknowledge the excellent technical assistance of Diana Woellner, Fränzi Creutzburg.

Conflict of interest All authors declare that they have no competing interests.

\section{References}

1. Jemal A, Bray F, Center MM, Ferlay J, Ward E, Forman D. Global cancer statistics. CA Cancer J Clin. 2011;61(2):69-90.

2. Aggarwal BB, Shishodia S, Sandur SK, Pandey MK, Sethi G. Inflammation and cancer: how hot is the link? Biochem Pharmacol. 2006;72(11):1605-21.

3. Kamangar F, Dawsey SM, Blaser MJ, Perez-Perez GI, Pietinen P, Newschaffer CJ, et al. Opposing risks of gastric cardia and noncardia gastric adenocarcinomas associated with Helicobacter pylori seropositivity. J Natl Cancer Inst. 2006;98(20):1445-52. 
4. Suerbaum S, Michetti P. Helicobacter pylori infection. N Engl J Med. 2002;347(15):1175-86.

5. Coussens LM, Werb Z. Inflammation and cancer. Nature (Lond). 2002;420(6917):860-7.

6. Kamangar F, Cheng C, Abnet CC, Rabkin CS. Interleukin-1B polymorphisms and gastric cancer risk: a meta-analysis. Cancer Epidemiol Biomarkers Prev. 2006;15(10):1920-8.

7. Peek RM Jr, Blaser MJ. Helicobacter pylori and gastrointestinal tract adenocarcinomas. Nat Rev Cancer. 2002;2(1):28-37.

8. Hitzler I, Sayi A, Kohler E, Engler DB, Koch KN, Hardt WD, et al. Caspase-1 has both proinflammatory and regulatory properties in Helicobacter infections, which are differentially mediated by its substrates IL-1beta and IL-18. J Immunol. 2012;188(8):3594-602.

9. El-Omar EM, Carrington M, Chow WH, McColl KE, Bream JH, Young HA, et al. Interleukin-1 polymorphisms associated with increased risk of gastric cancer. Nature (Lond). 2000; 404(6776):398-402.

10. El-Omar EM, Rabkin CS, Gammon MD, Vaughan TL, Risch HA, Schoenberg JB, et al. Increased risk of noncardia gastric cancer associated with proinflammatory cytokine gene polymorphisms. Gastroenterology. 2003;124(5):1193-201.

11. Tarlow JK, Blakemore AI, Lennard A, Solari R, Hughes HN, Steinkasserer A, et al. Polymorphism in human IL-1 receptor antagonist gene intron 2 is caused by variable numbers of an 86-bp tandem repeat. Hum Genet. 1993;91(4):403-4.

12. Graziano F, Ruzzo A, Santini D, Humar B, Tonini G, Catalano V, et al. Prognostic role of interleukin-1beta gene and interleukin-1 receptor antagonist gene polymorphisms in patients with advanced gastric cancer. J Clin Oncol. 2005;23(10):2339-45.

13. Stocker G, Ott K, Henningsen N, Becker K, Hapfelmeier A, Lordick F, et al. CyclinD1 and interleukin-1 receptor antagonist polymorphisms are associated with prognosis in neoadjuvanttreated gastric carcinoma. Eur J Cancer. 2009;45(18):3326-35.

14. Tahara T, Shibata T, Nakamura M, Yamashita H, Yoshioka D, Okubo M, et al. Effect of IL-1beta and TNF-alpha polymorphisms on the prognosis and survival of gastric cancer patients. Clin Exp Med. 2011;11(4):211-7.

15. Moehler M, Al-Batran SE, Andus T, Anthuber M, Arends J, Arnold D, et al. German S3-guideline "Diagnosis and treatment of esophagogastric cancer". Z Gastroenterol. 2011;49(4): 461-531.

16. Ferlay J, Shin HR, Bray F, Forman D, Mathers C, Parkin DM. Estimates of worldwide burden of cancer in 2008: GLOBOCAN 2008. Int J Cancer. 2010;127(12):2893-917.

17. McLean MH, El-Omar EM. Genetics of inflammation in the gastrointestinal tract and how it can cause cancer. Recent Results Cancer Res. 2011;185:173-83.

18. Dinarello CA. Immunological and inflammatory functions of the interleukin-1 family. Annu Rev Immunol. 2009;27:519-50.

19. Muerkoster S, Wegehenkel K, Arlt A, Witt M, Sipos B, Kruse ML, et al. Tumor stroma interactions induce chemoresistance in pancreatic ductal carcinoma cells involving increased secretion and paracrine effects of nitric oxide and interleukin-1beta. Cancer Res. 2004;64(4):1331-7.

20. Danis VA, Millington M, Hyland VJ, Grennan D. Cytokine production by normal human monocytes: inter-subject variation and relationship to an IL-1 receptor antagonist (IL-1Ra) gene polymorphism. Clin Exp Immunol. 1995;99(2):303-10.

21. Santtila S, Savinainen K, Hurme M. Presence of the IL-1RA allele $2(\mathrm{IL} 1 \mathrm{RN} * 2)$ is associated with enhanced IL-1beta production in vitro. Scand J Immunol. 1998;47(3):195-8.

22. Grabsch HI, Tan P. Gastric cancer pathology and underlying molecular mechanisms. Dig Surg. 2013;30(2):150-8.

23. Tamura G, Yin J, Wang S, Fleisher AS, Zou T, Abraham JM, Kong D, et al. E-Cadherin gene promoter hypermethylation in primary human gastric carcinomas. J Natl Cancer Inst. 2000;92(7):569-73.

24. Tahara T, Shibata T, Nakamura M, Yamashita H, Yoshioka D, Okubo M, et al. Effect of polymorphisms of IL-1beta and TNFalpha genes on $\mathrm{CpG}$ island hyper methylation (CIHM) in the nonneoplastic gastric mucosa. Mol Carcinog. 2011;50(11): $835-45$.

25. Xue H, Lin B, Ni P, Xu H, Huang G. Interleukin-1B and interleukin-1 RN polymorphisms and gastric carcinoma risk: a metaanalysis. J Gastroenterol Hepatol. 2010;25(10):1604-17.

26. Dean M, Carrington M, O’Brien SJ. Balanced polymorphism selected by genetic versus infectious human disease. Annu Rev Genomics Hum Genet. 2002;3:263-92.

27. Sellis D, Callahan BJ, Petrov DA, Messer PW. Heterozygote advantage as a natural consequence of adaptation in diploids. Proc Natl Acad Sci USA. 2011;108(51):20666-71.

28. Hellemann D, Larsson A, Madsen HO, Bonde J, Jarlov JO, Wiis $\mathrm{J}$, et al. Heterozygosity of mannose-binding lectin (MBL2) genotypes predicts advantage (heterosis) in relation to fatal outcome in intensive care patients. Hum Mol Genet. 2007; 16(24):3071-80.

29. Khor CC, Chapman SJ, Vannberg FO, Dunne A, Murphy C, Ling $\mathrm{EY}$, et al. A Mal functional variant is associated with protection against invasive pneumococcal disease, bacteremia, malaria and tuberculosis. Nat Genet. 2007;39(4):523-8.

30. Comings DE, MacMurray JP. Molecular heterosis: a review. Mol Genet Metab. 2000;71(1-2):19-31.

31. Flint J, Harding RM, Clegg JB, Boyce AJ. Why are some genetic diseases common? Distinguishing selection from other processes by molecular analysis of globin gene variants. Hum Genet. 1993;91(2):91-117.

32. Apte RN, Dotan S, Elkabets M, White MR, Reich E, Carmi Y, et al. The involvement of IL-1 in tumorigenesis, tumor invasiveness, metastasis and tumor-host interactions. Cancer Metastasis Rev. 2006;25(3):387-408.

33. Apte RN, Voronov E. Is interleukin-1 a good or bad 'guy' in tumor immunobiology and immunotherapy? Immunol Rev. 2008;222:222-41.

34. Dinarello CA. Why not treat human cancer with interleukin-1 blockade? Cancer Metastasis Rev. 2010;29(2):317-29. 